\title{
Órteses para o paciente com osteoartrite do polegar: o que os terapeutas ocupacionais no Brasil indicam?*
}

\section{Orthoses for patients with thumb osteoarthritis: what occupational therapists in Brazil indicate?}

\author{
Pedro Henrique T. Q. de Almeida ${ }^{1}$, Tatiana Barcelos Pontes ${ }^{2}$, Jackelline R. Lopes Rossi ${ }^{3}$, \\ Clarissa C. dos Santos-Couto-Paz ${ }^{4}$, Joy C. MacDermid ${ }^{5}$, João Paulo Chieregato Matheus ${ }^{6}$
}

http://dx.doi.org/10.11606/issn.2238-6149.v27i3p289-296

\begin{abstract}
Almeida PHTQ, Pontes TB, Rossi JRL, Santos-Couto-Paz CC, Macdermid JC, Matheus JPC. Órteses para o paciente com osteoartrite do polegar: o que os terapeutas ocupacionais no Brasil indicam? Rev Ter Oucp Univ São Paulo. 2016 set.-dez.;27(3):289-96.
\end{abstract}

RESUMO: Órteses são consideradas a primeira linha de atuação para o tratamento de disfunções causadas pela osteoartrite $(\mathrm{OA})$ da articulação carpometacárpica do polegar. Entretanto, não existem estudos que investiguem as preferências e indicações de órteses por profissionais brasileiros. Objetivo: Identificar os modelos de órteses indicados por terapeutas ocupacionais para pacientes com OA do polegar, bem como as barreiras encontradas para o uso deste recurso. Métodos: Estudo exploratório transversal, realizado através de questionário eletrônico com terapeutas ocupacionais brasileiros. Os dados obtidos foram analisados por meio da distribuição, frequência e porcentagem das respostas. Resultados: Participaram da pesquisa 52 terapeutas ocupacionais de todo o Brasil, atuando junto a pacientes reumáticos. A maioria das indicações foi de órteses sob medida (81\%), envolvendo mais de uma articulação (83,7\%). Políticas institucionais e ausência de materiais foram as principais dificuldades para $48,1 \%$ dos participantes. Conclusão: Aindicação de órteses para pacientes com OA se faz presente na prática clínica de terapeutas ocupacionais e torna-se importante o incentivo a estratégias que permitam o aperfeiçoamento e difusão de ações de reabilitação junto a esta população.

DESCRITORES: Osteoartrite; Polegar; Aparelhos ortopédicos; Órteses; Terapia ocupacional; Equipamentos de auto-ajuda; Brasil.
PHTQ, Pontes TB, Rossi JRL, Santos-Couto-Paz CC, Macdermid JC, Matheus JPC. Orthoses for patients with thumb osteoarthritis: what occupational therapists in Brazil indicate? Rev Ter Oucp Univ São Paulo. 2016 Sept.-Dec.;27(3):289-96.

\begin{abstract}
Orthoses are the first line of action for the treatment of dysfunctions caused by osteoarthritis (OA) of the carpometacarpal joint of the thumb. However, there are no studies that investigate the preferences and orthotics prescriptions made by Brazilian health professionals. Objective: To identify orthotic designs indicated by occupational therapists for patients with thumb OA and the barriers encountered to use these devices. Methods: Crosssectional, exploratory study conducted by electronic questionnaire with Brazilian occupational therapists. Data were analyzed using the distribution, frequency and percentage of responses. Results: 52 Brazilian occupational therapists who treated patients with rheumatic diseases participated in this study. Most of the participants indicated custom-made models ( $81 \%$ ), involving more than one joint (83.7\%). Institutional policies and lack of materials were the main barriers for $48.1 \%$ of the participants. Conclusion: The prescription of orthoses for patients with OA is present in the clinical practice of occupational therapists, and it is important to encourage strategies to the development and dissemination of rehabilitation actions for this population.
\end{abstract}

KEYWORDS: Osteoarthritis; Thumb; Orthotic devices; Occupational therapy; Self-help-devices; Brazil.

\footnotetext{
*Este trabalho é parte do projeto de pesquisa de doutorado intitulado "Influência do uso de órteses para osteoartrite do polegar: análise cinemática e funcional” do Programa de Pós-Graduação em Ciências e Tecnologias em Saúde da Universidade de Brasília. Pesquisa financiada pelo CNPq.

1. Professor Assistente, Curso de Terapia Ocupacional, Universidade de Brasília. Doutorando em Ciências e Tecnologias em Saúde pela Universidade de Brasília. Email: pedroalmeida.to@gmail.com.

2. Professora Adjunta, Curso de Terapia Ocupacional, Universidade de Brasília. Doutora em Saúde da Criança e do Adolescente pela FMRP-USP. Email: tatiana.pontes@gmail.com.

3. Terapeuta Ocupacional, Universidade de Brasília. Email: jackellinerl@gmail.com.

4. Professora Adjunta, Curso de Fisioterapia, Universidade de Brasília. Programa de Pós-Graduação em Ciências e Tecnologias em Saúde - UnB. Doutora em Neurociências pela UFMG. Email: clarissacardososcp@gmail.com.

5. Professor and Assistant Dean, School of Rehabilitation Sciences, McMaster University, Hamilton, Ontario. Research Coordinator, WSIB Upper Limb Speciality Clinic, St. Joseph's Health Centre - London, Ontario. Email: macderj@mcmaster.ca.

6. Professor Adjunto, Curso de Fisioterapia, Universidade de Brasília. Programa de Pós-Graduação em Ciências e Tecnologias em Saúde - UnB. Doutor em Ciências Médicas pela FMRP-USP. Email: jpcmatheus@unb.br.

Endereço para correspondência - Pedro H.T.Q. de Almeida. Faculdade de Ceilândia - Universidade de Brasília - Centro Metropolitano, conjunto A, lote 01, Brasília, DF. CEP: 72220-275.
} 


\section{INTRODUÇÃO}

A osteoartrite (OA) é uma doença que acomete o sistema musculoesquelético, caracterizada pela degeneração focal de cartilagem hialina em articulações sinoviais, com hipertrofia óssea e consequente espessamento da cápsula articular, ocasionada pelo desequilíbrio do mecanismo de síntese e degradação da cartilagem articular ${ }^{1,2}$.

A OA é considerada a doença articular crônica de maior prevalência entre a população, sendo a primeira causa de dor e limitação funcional entre adultos e idosos ${ }^{2-6}$. Estima-se a OA afete $15 \%$ da população adulta no mundo, com prevalência superior a $66 \%$ para mulheres acima de 55 anos $^{3}$. No Brasil, a prevalência de osteoartrite entre a população maior de 60 anos varia entre $9,4 \%$ a $39,6 \%$, sendo maior o acometimento de joelhos e mãos ${ }^{7}$.

Dentre as diversas manifestações da doença, o acometimento das mãos pela $\mathrm{OA}$ acarreta importantes restrições funcionais, como presença de dor intermitente, associada ao movimento e/ou sustentação de cargas em qualquer atividade que envolva a preensão e manuseio de objetos $^{5}$. Além das limitações ocupacionais associadas a dor, a rigidez articular e restrições de mobilidade dificultam ou impossibilitam a execução de atividades laborais, de manutenção do lar, recreação e autocuidado ${ }^{8}$.

Observa-se que a articulação carpometacárpica (CMC) do polegar figura entre as mais acometidas, correspondendo a $8 \%$ de todos os casos de OA diagnosticados $^{1}$. Embora de menor prevalência quando comparada a manifestações da doença em joelhos ${ }^{2}$, articulações metacarpofalangeanas (MF) e interfalangeanas distais (IFD) $)^{2,3,6,9}$, o acometimento do polegar ocasiona severas restrições funcionais devido à limitação da função preensora das mãos ${ }^{2}$, essencial ao desempenho e participação em diversas atividades.

Considerando-se o caráter crônico da $\mathrm{OA}$ e seu impacto sobre várias áreas de desempenho do paciente ${ }^{10}$, consensos emitidos por entidades internacionais e também pela Sociedade Brasileira de Reumatologia ${ }^{11}$ preconizam de forma unânime o acompanhamento por equipe multiprofissional ${ }^{9}$, na qual o terapeuta ocupacional atua de forma a habilitar o engajamento do paciente em ocupações. Tal intervenção consiste na combinação de exercícios, técnicas de educação do paciente e uso de órteses para promover suporte e proteção articular ${ }^{6}$.

$\mathrm{O}$ uso de órteses para pacientes com OA objetiva o controle da dor e redução da rigidez matinal, o suporte mecânico para articulações favorecendo a movimentação ${ }^{3}$, sobretudo durante a realização de atividades em que a combinação entre alinhamento articular, imobilização e aplicação de forças de tração é necessária ${ }^{12,13}$, tais como costurar, tocar um instrumento musical, escrever, digitar no computador ou em telefones celulares ${ }^{10}$.

Observa-se que, apesar da uniformidade de objetivos para o uso de órteses junto a pacientes com OA do polegar, o caráter único desta articulação constitui um desafio, uma vez que a órtese deverá promover estabilização articular sem impedir sua movimentação ${ }^{3}$. Desta forma, uma ampla variedade de modelos e estratégias de tratamento por meio de órteses foi desenvolvida ao longo das últimas duas décadas, resultando em dezenas de dispositivos ${ }^{6,14,15}$.

Tal gama de possibilidade de intervenção é tida como um dos fatores que levam a variações na prática profissional ${ }^{4}$, dificultando a avaliação da eficácia de procedimentos terapêuticos específicos ${ }^{2},{ }^{3}$. Estudos conduzidos junto a terapeutas e pacientes norte americanos ${ }^{4}$ demonstraram variações significativas tanto para a escolha da órtese, quanto para as estratégias de raciocínio clínico utilizadas durante o tratamento da OA do polegar. Tais práticas resultaram em abordagens terapêuticas que, muitas vezes diferiam dos objetivos e expectativas dos pacientes, ocasionando abandono do tratamento e dos dispositivos prescritos ${ }^{10}$.

Tendo em vista a escassez de estudos sobre práticas profissionais de terapeutas ocupacionais atuando junto a pacientes reumatológicos no Brasil e as diferenças dos contextos sociais e políticos envolvendo a prescrição de dispositivos de tecnologia assistiva em países em desenvolvimento ${ }^{16,17}$, o presente estudo buscou estabelecer o perfil do uso de órteses na clínica de terapia ocupacional junto a pacientes com OA do polegar em nosso país, identificando preferências dos tipos de órteses, materiais, modelos e articulações envolvidas, assim como barreiras e dificuldades enfrentadas para seu uso junto a esta população.

\section{METODOLOGIA}

Este estudo teve início a partir de uma pesquisa de doutorado conduzida junto ao Programa de Pós-Graduação em Ciências e Tecnologias em Saúde da Universidade de Brasília, que teve por objetivo verificar a influência de órteses sobre a função manual de pacientes com $\mathrm{OA}$ do polegar.

O levantamento dos dados referentes à prescrição de órteses por terapeutas ocupacionais brasileiros foi conduzido por meio de questionário eletrônico elaborado através da plataforma Google Docs ${ }^{\circledR}$. O questionário foi 
composto por 40 questões, divididas três seções abordando os temas Formação e Atuação Profissional, Prática Junto à Pacientes Reumáticos e Uso de Órteses durante o tratamento destes pacientes.

As questões referentes à Formação e Atuação Profissional envolveram sete perguntas fechadas sobre graduações adicionais à formação em terapia ocupacional, pós-graduação (Lato Sensu e Stricto Sensu), área de especialização e atuação, além do tempo de atuação e população atendida. A Prática Junto à Pacientes Reumáticos foi avaliada por meio de cinco questões, identificando $\mathrm{o}$ atendimento a pacientes com doenças reumáticas específicas e tratamento de pessoas com osteoartrite do polegar, bem como uso ou não de órteses enquanto recurso terapêutico para o tratamento desta condição.

As questões voltadas para o uso de órteses na OA do polegar compuseram a maior parte do questionário, correspondendo a 28 perguntas. Foram investigados o tipo de órteses prescritas (se pré-fabricadas ou confeccionadas sob medida), material de fabricação e indicação de modelos específicos. Além de questões sobre quais órteses os terapeutas ocupacionais indicam, as barreiras e dificuldades enfrentadas durante esta intervenção foram avaliadas por meio de uma seção final, composta por questões relacionadas às dificuldades enfrentadas para a indicação e uso de órteses, contendo exemplos de barreiras descritas em estudos similares previamente realizados ${ }^{4}$.

As respostas referentes às barreiras e dificuldades foram organizadas em quatro categorias, divididas entre Políticas Institucionais (referentes aos sistemas de encaminhamento de pacientes, processos de referência e contra referência, organização institucional e especificidades da rede de atenção à saúde), Ausência de Equipamentos (materiais permanentes necessários para a confecção de órteses, como panelas, tesouras, sopradores de ar), Ausência de Materiais de Consumo (termoplástico, Velcro®, Neoprene) e Ausência de Conhecimentos Específicos sobre órteses. Comentários realizados pelos participantes foram incluídos na análise dos dados.

Para elaboração das questões sobre uso de órteses, os dois primeiros autores conduziram de forma independente uma pesquisa bibliográfica em diferentes bases de dados eletrônicas, livros e materiais instrucionais, a fim de disponibilizar aos participantes descrições do maior número possível de modelos. Foram incluídos artigos científicos, descrições e instruções para a elaboração de modelos de órteses utilizados junto a pacientes com OA do polegar. Além disto, foram incluídas órteses comerciais, por meio da pesquisa em catálogos físicos e eletrônicos de fabricantes.
A primeira etapa da pesquisa resultou em 25 modelos distintos, que foram adquiridos ou confeccionados pelos autores. Os modelos foram fotografados em três planos e suas imagens inseridas no formulário eletrônico (Figura 1), acompanhadas da descrição dos modelos de acordo com as recomendações da Sociedade Americana de Terapeutas da Mão ${ }^{18}$.

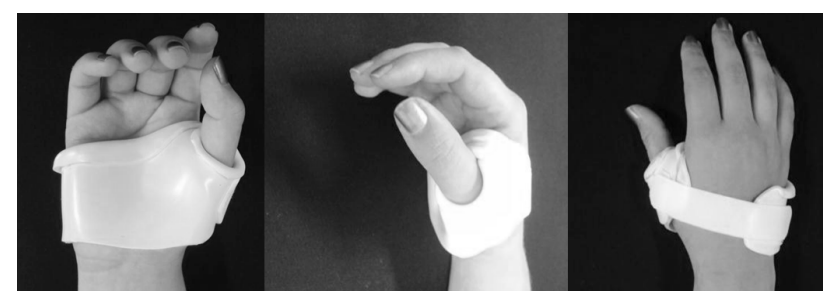

Figura 1 - Planos utilizados para fotografias das órteses

Um link para o questionário foi encaminhado através de e-mail para terapeutas ocupacionais cadastrados nas regionais 1 a 11 do Conselho Regional de Fisioterapia e Terapia Ocupacional (CREFITO) e através de sites e grupos de e-mail de associações profissionais. O convite foi enviado por três vezes entre os meses de Julho e Setembro de 2014. Foram excluídos questionários incompletos, preenchidos por graduandos ou profissionais que exerciam outra profissão.

Dados do Conselho Federal de Fisioterapia e Terapia Ocupacional (COFFITO) indicam a existência de 16.711 terapeutas ocupacionais cadastrados em Setembro de 2015. Entretanto, não existem dados referentes ao número de terapeutas ocupacionais atuando especificamente junto a pacientes com doenças reumáticas, o que inviabiliza a estimativa de um tamanho amostral mínimo.

O projeto foi aprovado pelo Comitê de Ética em Pesquisa da instituição proponente do estudo, através parecer 392.890/2013. Os participantes foram orientados a preencher o Termo de Consentimento Livre e Esclarecido digital, anteriormente ao preenchimento do questionário.

Os dados quantitativos obtidos foram analisados por meio da distribuição, frequência e porcentagem das respostas. Uma vez que o questionário incluiu questões de múltipla escolha, algumas respostas apresentaram porcentagens totais superiores a $100 \%$. Para todas as análises foi utilizado o software SPSS, versão 20.0.

\section{RESULTADOS}

\section{Caracterização da amostra}

Responderam ao questionário 68 terapeutas ocupacionais de todo o país. Para a análise dos dados desta 
pesquisa, foram consideradas apenas respostas fornecidas por terapeutas que atuam junto a pacientes com doenças reumatológicas, sendo a amostra final composta por 52 profissionais.

A maioria dos participantes foi composta por terapeutas ocupacionais do sexo feminino, com tempo de atuação profissional de até cinco anos. No total, $65 \%$ dos participantes possuíam pós-graduação lato sensu como maior qualificação profissional, sendo que mais da metade destes declararam possuir especialização em terapia da mão e reabilitação do membro superior.

A classificação dos respondentes quanto à região de atuação indicou a concentração de 37 terapeutas participantes entre as regiões sul e sudeste, correspondendo a $72 \%$ da amostra total. Todas as regiões do país foram contempladas com ao menos um terapeuta ocupacional respondente (Tabela 1).

Tabela 1 - Características Demográficas dos Participantes

\begin{tabular}{|c|c|}
\hline & n (\%) \\
\hline \multicolumn{2}{|l|}{ Sexo } \\
\hline Masculino & $5(9,6)$ \\
\hline Feminino & $47(90,4)$ \\
\hline Total & $52(100)$ \\
\hline \multicolumn{2}{|l|}{ Tempo de Atuação Profissional } \\
\hline 0 a 5 anos & $20(38,5)$ \\
\hline 6 a 10 anos & $14(26,9)$ \\
\hline 11 a 15 anos & $8(15,4)$ \\
\hline Superior a 15 anos & $10(19,2)$ \\
\hline \multicolumn{2}{|l|}{ Maior nível de Qualificação Profissional } \\
\hline Graduação & $3(5,8)$ \\
\hline Pós-Graduação stricto senso (especialização) & $34(65,4)$ \\
\hline Mestrado & $11(21,2)$ \\
\hline Doutorado & $4(7,6)$ \\
\hline \multicolumn{2}{|l|}{ Áreas de especialização Lato Sensu * } \\
\hline Terapia da Mão e Reabilitação do Membro Superior & $30(57,7)$ \\
\hline Terapia Ocupacional em Reabilitação Física & $8(15,4)$ \\
\hline Terapia Ocupacional Neurologia & $7(13,5)$ \\
\hline Terapia Ocupacional em Reumatologia & $4(7,7)$ \\
\hline Terapia Ocupacional Ortopedia & $4(7,7)$ \\
\hline Outras & $4(7,7)$ \\
\hline \multicolumn{2}{|l|}{ Região de Atuação } \\
\hline Sudeste & $28(53,8)$ \\
\hline Sul & $9(17,3)$ \\
\hline Centro-Oeste & $8(15,4)$ \\
\hline Nordeste & $6(11,5)$ \\
\hline Norte & $1(1,9)$ \\
\hline
\end{tabular}

* Questão de múltipla escolha: a porcentagem total pode exceder 100\%. 


\section{Uso de Órteses para tratamento da Osteoartrite do Polegar}

A indicação de órteses para pacientes com OA do polegar foi uma modalidade de intervenção utilizada por $94 \%$ dos participantes. A maioria dos profissionais consultados optou por modelos confeccionados sob medida para o paciente $(81 \%)$, sendo que apenas sete terapeutas indicavam tanto órteses pré-fabricadas quanto sob medida. Nenhum terapeuta afirmou indicar apenas órteses pré-fabricadas.

Dentre os materiais utilizados para confecção das órteses, o termoplástico de baixa temperatura foi indicado como mais utilizado por $83 \%$ dos participantes, seguido pelo neoprene (23\%) e por termoplásticos de alta temperatura (Tabela 2).

Tabela 2 - Órteses e materiais utilizados

\begin{tabular}{l|c}
\hline & n (\%) \\
\hline Uso e Indicação de Órteses & $0(0)$ \\
\hline Indica Órteses Pré-Fabricadas & $42(81)$ \\
\hline Indica Órteses Sob Medida & $7(13)$ \\
\hline Indica Ambos os tipos & $4(6)$ \\
\hline Não Utiliza Órteses & \\
\hline $\begin{array}{l}\text { Materiais utilizados para confecção de } \\
\text { órteses* }\end{array}$ & $43(83)$ \\
\hline Termoplásticos de baixa temperatura & $12(23)$ \\
\hline Neoprene & $8(15)$ \\
\hline Termoplásticos de alta temperatura & $3(6)$ \\
\hline Outros (Couro, lona, metais) & $1(2)$ \\
\hline Não sabe &
\end{tabular}

* Questão de múltipla escolha: a porcentagem total pode exceder $100 \%$.

Em relação à imobilização das articulações adjacentes à articulação carpometacárpica do polegar, $84 \%$ dos terapeutas ocupacionais preferiram designs de órteses que imobilizassem ao menos a articulação metacarpofalangeana (MCF) juntamente com a articulação CMC, seguido por modelos longos, que incluíam também a articulação do punho. Modelos curtos, com menor restrição de movimento, foram indicados por apenas 47\% dos participantes. O Gráfico 1 ilustra os resultados desta etapa.

No Gráfico 1: Os modelos de órteses apresentados foram divididos de acordo com seu local de aplicação e número de articulações envolvidas (Punho, Carpometacárpica e Metacarpofalangeana do primeiro dedo). Foi observada a preferência dos participantes por modelos que incluíam ao menos uma articulação adjacente. Questão de múltipla escolha: a soma das porcentagens obtidas por ser superior a $100 \%$

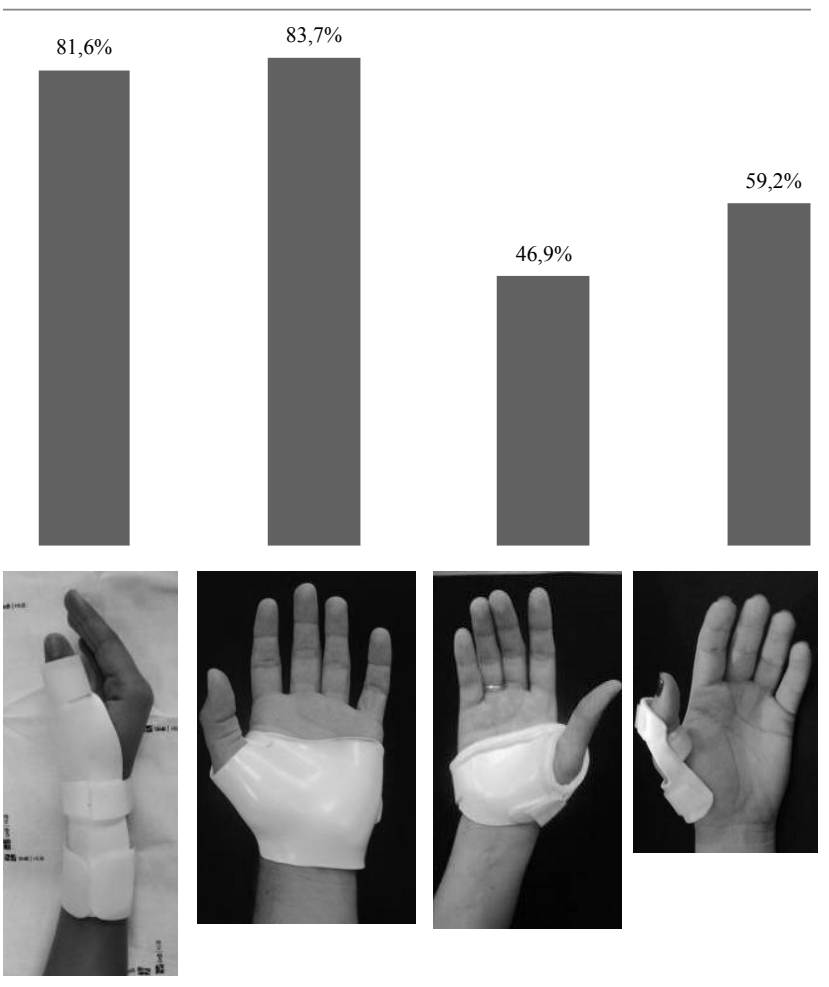

Gráfico 1 - Indicação de modelos de órteses

\section{Dificuldades e barreiras para o uso de órteses}

Os dados referentes às dificuldades para a o uso de órteses junto a pacientes com osteoartrite de polegar indicaram maior prevalência de queixas relacionadas a políticas institucionais, consideradas por $48 \%$ dos terapeutas respondentes como principal barreira. Dificuldades referentes ao processo de encaminhamento de pacientes, existência de setores específicos na rede de saúde ou na própria instituição e alto fluxo atendimentos foram apontados como principais dificuldades para o uso de órteses junto a esta população.

A ausência de materiais de consumo para confecção das órteses foi relatada como principal dificuldade por $25 \%$ da amostra. A Tabela 3 apresenta os resultados referentes a esta etapa. 
Almeida PHTQ, et al. Órteses para o paciente com osteoartrite do polegar. Rev Ter Ocup Univ São Paulo. 2016 set./dez.;27(3):289-96.

Tabela 3 - Dificuldades e barreiras para o uso de órteses

\begin{tabular}{l|c}
\hline & n (\%) \\
\hline Dificuldades/Barreiras & \\
\hline Políticas Institucionais & $25(48.1)$ \\
\hline Ausência de Material de Consumo & $13(25)$ \\
\hline Ausência de Equipamentos & $7(13.5)$ \\
\hline Ausência de Conhecimentos Específicos & $7(13.5)$ \\
\hline
\end{tabular}

\section{DISCUSSÃO}

Aindicação de órteses confeccionadas sobre medida, em termoplásticos de baixa temperatura foi predominante entre a amostra consultada. Estudo realizado por Colditz ${ }^{15}$, aponta que a introdução deste material o final dos anos 1970 modificou consideravelmente a fabricação de órteses por terapeutas ocupacionais, permitindo a confecção de dispositivos altamente personalizados ${ }^{15}$.

A indicação exclusiva de órteses sob medida contraria tendências observadas em outros países: em um estudo conduzido com 627 terapeutas ocupacionais e fisioterapeutas norte-americanos, O'Brien e $\mathrm{McGaha}^{4}$ observaram que a maioria dos profissionais prescrevia ao menos um modelo pré-fabricado e um modelo sob medida 4 . Embora não existam estudos sobre esta temática no Brasil, a maior disponibilidade de dispositivos e materiais préfabricados em países da América do Norte e Europa e a escassez destes recursos em países em desenvolvimento como o Brasil ${ }^{16,17}$ pode justificar as diferenças encontradas.

Em nosso estudo, a maior parte dos terapeutas indicou preferência por órteses que envolvessem as articulações CMC e MCF do polegar, seguidas por modelos longos, envolvendo o punho. Órteses curtas apresentaram o menor número de indicações, sendo a distribuição das respostas similar aos resultados de O'Brien e $\mathrm{McGaha}^{4}$.

Embora numerosas pesquisas ${ }^{15,19-21}$ sugiram melhoras funcionais através de órteses curtas, envolvendo muitas vezes apenas a articulação $\mathrm{CMC}$ do polegar e promovendo estabilização articular por meio de materiais maleáveis, como o Neoprene ${ }^{20,21}$, o uso de órteses que promovam maior restrição articular ainda é predominante em pesquisas ${ }^{14,19,21-23}$ e na prática clínica. Apesar das evidências recentes sugerirem o uso de órteses curtas, a diretriz publicada pela European League Against Rheumatism (EULAR) em 2007 indica o uso de órteses longas para a OA do polegar.

A diferença entre o preconizado pela literatura e a prática clínica não é exclusividade do cenário da terapia ocupacional brasileira, uma vez que padrões de prescrições de órteses semelhantes foram observados entre terapeutas dos Estados Unidos e Canadá, que indicam a constante necessidade de atualização como um dos principais desafios da profissão ${ }^{4}$.

Dentre as dificuldades elencadas pelos participantes, as políticas institucionais consistiram a maior barreira para a indicação de órteses para pacientes com OA do polegar. Tal dificuldade pode englobar pontos relacionados ao processo de encaminhamento de pacientes, associado à organização institucional e da rede de saúde. Embora a prescrição e dispensação de órteses seja prevista pelo Sistema Único de Saúde e faça parte das intervenções gratuitas oferecidas à população brasileira, observa-se que tais serviços nem sempre estão disponíveis. Em um estudo conduzido com profissionais responsáveis pela dispensação de tecnologias assistivas em três cidades do interior de São Paulo, Caro et al. ${ }^{24}$ observaram a ausência de serviços especializados e necessidade de encaminhamento de pacientes para grandes centros fora da área de abrangência da regional de saúde da qual o paciente é proveniente.

A combinação da escassez de serviços especializados com a necessidade de encaminhamento de pacientes para centros de dispensação de tecnologias assistivas ocasiona aumento do tempo de espera e reduzida efetividade destes serviços $^{24}$, o que leva a sobrecarga dos profissionais de serviços especializados e a não utilização das habilidades dos profissionais nos demais serviços de saúde.

A ausência de matérias de consumo, sobretudo termoplásticos de baixa temperatura, foi também indicada com barreira para o uso de órteses junto à população com OA do polegar. Embora este material tenha sido apontado como preferencial para a confecção de órteses por $83 \%$ dos terapeutas respondentes, similar à preferência encontrada por Agnelli e Toyoda ${ }^{25}$, o alto custo do material, associado a dificuldades do processo de importação, pode ser um fator que contribui para sua escassez em serviços de saúde e centros de dispensação de órteses ${ }^{25}$.

\section{Limitações do Estudo}

Apesar dos esforços dispendidos pelos autores e colaboradores para a divulgação desta pesquisa entre terapeutas ocupacionais, a taxa de resposta obtida no presente estudo mostrou-se limitada para uma pesquisa de âmbito nacional. Dados da Sociedade Brasileira de Terapia da Mão e do Membro Superior (SBTM) mostram que existem 71 terapeutas ocupacionais de todo o país inscritos nesta associação que historicamente está relacionada a profissionais que utilizam órteses como 
recurso terapêutico, número pouco superior ao tamanho amostral do presente estudo. Entretanto, acredita-se que muitos profissionais não sejam membros da SBTM, desta forma, a inexistência de informações referentes a área de atuação dos terapeutas ocupacionais não permitiu estimar a taxa de resposta obtida neste estudo com precisão.

No questionário de coleta de dados, a ausência de questões que pudessem identificar o contexto de prática profissionais dos terapeutas ocupacionais participantes foi outro fator limitador, uma vez que impossibilita uma discussão mais ampla a respeito das diferenças encontradas entre os serviços públicos e privados e o impacto de políticas públicas para a prescrição e dispensação de órteses nestes espaços.

Apesar disto, este estudo contribuiu para uma maior compreensão sobre a indicação do tipo de órteses para OA por terapeutas ocupacionais no Brasil, obtendo informações de profissionais de todas as cinco regiões do país.

\section{CONCLUSÕES}

Os resultados obtidos indicaram preferência dos terapeutas ocupacionais participantes do estudo por órteses sob medida, confeccionadas em material termoplástico e envolvendo as articulações do punho, carpometacárpica e metacarpofalangeana do polegar. Além disto, as dificuldades apontadas pelos profissionais sugerem que a maior parte das barreiras para o uso de órteses é originária da organização da rede de atenção à saúde.

A baixa preferência por órteses que estabilizem exclusivamente a articulação $\mathrm{CMC}$ do polegar pode indicar um distanciamento da prática adotada em clínicas e centros de reabilitação dos resultados de pesquisas científicas sobre a temática.

\section{Impacto para a prática clínica}

Por meio dos resultados obtidos puderam-se identificar pontos relevantes da prática com órteses da terapia ocupacional brasileira durante o tratamento do paciente com osteoartrite do polegar. A avaliação e reflexão sobre a necessidade de ajustes à atenção prestada a esta população deve ser enfatizada por profissionais, gestores de saúde e instituições de formação em saúde.

Agradecimentos: Os autores gostariam de agradecer ao Conselho Nacional de Desenvolvimento Científico e Tecnológico (CNPq) Programa Ciências Sem Fronteiras pelo apoio financeiro para realização desta pesquisa e a Prof ${ }^{\text {a }}$ Dr $^{\mathrm{a}}$ Iracema S.V. Ferrigno pela revisão e auxílio para a divulgação do questionário. Agradecemos também a todas as regionais do CREFITO que colaboraram com a divulgação desta pesquisa, em especial ao CREFITO da $11^{\text {a }}$ Região.

Contribuição dos autores: Pedro H.T.Q. de Almeida: Idealização e concepção metodológica da pesquisa. Coleta e análise dos dados, redação do artigo; Tatiana B. Pontes: Idealização e concepção metodológica da pesquisa. Coleta e análise dos dados, redação e revisão do artigo; Jackelline R. L. Rossi: Coleta e análise dos dados, redação do artigo; Clarissa C. dos Santos-Couto-Paz: Idealização e concepção metodológica da pesquisa. Análise dos dados, redação e revisão do artigo; Joy C. MacDermid: Idealização e concepção metodológica; Análise dos dados; João Paulo C. Matheus: Idealização e concepção metodológica da pesquisa. Coleta e análise dos dados, redação e revisão do artigo.

\section{REFERÊNCIAS}

1. Dahaghin S, Bierma-Zeinstra SM, Ginai AZ, Pols HA, Hazes JM, Koes BW. Prevalence and pattern of radiographic hand osteoarthritis and association with pain and disability (the Rotterdam study). Ann Rheum Dis. 2005;64:682-7. doi: 10.1136/ard.2004.023564.
2. Kloppenburg M. Hand osteoarthritis-nonpharmacological and pharmacological treatments. Nature Rev Rheumatol. 2014;10:242-51. doi: 10.1038/nrrheum.2013.214.

3. Bertozzi L, Valdes K, VantiC, Negrini S, PillastriniP, Villafane $\mathrm{JH}$. Investigation of the effect of conservative interventions 
Almeida PHTQ, et al. Órteses para o paciente com osteoartrite do polegar. Rev Ter Ocup Univ São Paulo. 2016 set./dez.;27(3):289-96.

in thumb carpometacarpal osteoarthritis: systematic review and meta-analysis. Disabil Rehabil. 2015;37(22):2025-43. doi: 10.3109/09638288.2014.996299.

4. O'Brien VH, McGaha JL. Current practice patterns in conservative thumb CMC joint care: survey results. J Hand Ther. 2014;27:14-22. doi: 10.1016/j.jht.2013.09.001.

5. O'Brien VH, Giveans MR. Effects of a dynamic stability approach in conservative intervention of the carpometacarpal joint of the thumb: a retrospective study. J Hand Ther. 2013;26:44-52. doi: 10.1016/j.jht.2012.10.005.

6. Kjeken I, Smedslund G, Moe RH, Slatkowsky-Christensen B, Uhlig T, Hagen KB. Systematic review of design and effects of splints and exercise programs in hand osteoarthritis. Arthritis Care \& Research. 2011; 63: 834-48. DOI: 10.1002/acr.20427.

7. Miranda VS, Decarvalho VB, Machado LA, Dias JM. Prevalence of chronic musculoskeletal disorders in elderly Brazilians: a systematic review of the literature. BMC Musculoskelet Disord. 2012;13:82. doi: 10.1186/1471-2474-13-82.

8. Hammond A. Joint protection. In: Goodacre L, McArthur M, editors. Rheumatology practice in occupational therapy. Oxford: Willey-Blackwell; 2013. p.111-32.

9. Hochberg MC, Altman RD, April KT, et al. American College of Rheumatology 2012 recommendations for the use of nonpharmacologic and pharmacologic therapies in osteoarthritis of the hand, hip, and knee. Arthritis Care Res (Hoboken). 2012;64:465-74. doi: 10.1002/acr.21596.

10. Frouzakis R, Herren DB, Marks M. Evaluation of expectations and expectation fulfillment in patients treated for trapeziometacarpal osteoarthritis. J Hand Surg Am. 2015;40:483-90. doi: 10.1016/j.jhsa.2014.10.066.

11. Coimbra IB, Pastor EH, Greve J, et al. Consenso brasileiro para o tratamento da osteoartrite (artrose). Rev Bras Reumatol. 2002;42:371-4.

12. McDonald HN, Dietrich T, Townsend A, Li LC, Cox S, Backman CL. Exploring occupational disruption among women after onset of rheumatoid arthritis. Arthritis Care Res (Hoboken). 2012;64:197-205. doi: 10.1002/acr.20668.

13. Bansback N, Zhang W, Walsh D, et al. Factors associated with absenteeism, presenteeism and activity impairment in patients in the first years of RA. Rheumatology (Oxford). 2012;51:375-84. doi: 10.1093/rheumatology/ker385.

14. Neumann DA, Bielefeld T. The carpometacarpal joint of the thumb: stability, deformity, and therapeutic intervention. J Orthop Sports Phys Ther. 2003;33:386-99. doi: 10.2519/ jospt.2003.33.7.386.

Recebido em: 17.11 .15

Aceito em: 10.10 .16
15. Colditz JC. The biomechanics of a thumb carpometacarpal immobilization splint: design and fitting. J Hand Ther. 2000;13:228-35. doi: 10.1016/S0894-1130(00)80006-X.

16. Borg J, Ostergren PO. Users' perspectives on the provision of assistive technologies in Bangladesh: awareness, providers, costs and barriers. Disabil Rehabil Assist Technol. 2015;10:301-8. doi: 10.3109/17483107.2014.974221.

17. Rios A, Miguel Cruz A, Guarin MR, Caycedo Villarraga PS. What factors are associated with the provision of assistive technologies: the Bogota D.C. case. Disabil Rehabil Assist Technol. 2014;9:432-44. doi: 10.3109/17483107.2014.936053.

18. American Society of Hand Therapists (ASHT). Splint classification system. Chicago; 1992

19. Maddali-Bongi S, Del Rosso A, Galluccio F, Sigismondi F, Matucci-Cerinic M. Is an intervention with a custommade splint and an educational program useful on pain in patients with trapeziometacarpal joint osteoarthritis in a daily clinical setting? Int J Rheum Dis. 2016;19(8):773-80. doi: 10.1111/1756-185X.12318.

20. Hermann M, Nilsen T, Eriksen CS, Slatkowsky-Christensen B, Haugen IK, Kjeken I. Effects of a soft prefabricated thumb orthosis in carpometacarpal osteoarthritis. Scand J Occup Ther. 2014;21:31-9. Doi: 10.3109/11038128.2013.851735.

21. Sillem H, Backman CL, Miller WC, Li LC. Comparison of two carpometacarpal stabilizing splints for individuals with thumb osteoarthritis. J Hand Ther. 2011;24:216-25; quiz 126; discussion 227-30. doi: 10.1016/j.jht.2010.12.004.

22. Gomes Carreira AC, Jones A, Natour J. Assessment of the effectiveness of a functional splint for osteoarthritis of the trapeziometacarpal joint on the dominant hand: a randomized controlled study. J Rehabil Med. 2010;42:46974. doi: 10.2340/16501977-0542.

23. Ford M, McKee P, Szilagyi M. A hybrid thermoplastic and neoprene thumb metacarpophalangeal joint orthosis. J Hand Ther. 2004;17:64-8. doi: 10.1197/j.jht.2003.10.009.

24. Caro CC, Faria PS, Bombarda TB, Ferrigno ISV, Palhares MS. A dispensação de órteses, próteses e meios auxiliares de locomoção (OPM) no Departamento Regional de Saúde da $3^{\text {a }}$ Região do Estado de São Paulo. Cad Ter Ocup UFSCar. 2014;22(3):521-9. doi: 10.4322\%2Fcto.2014.073.

25. Agnelli LB, Toyoda CY. Estudo de materiais para a confecção de órteses e sua utilização prática por Terapeutas Ocupacionais no Brasil. Cad Ter Ocup UFSCar. 2003;11. Dsisponível em: http://www.cadernosdeterapiaocupacional. ufscar.br/index.php/cadernos/article/view/194/149. 